\title{
Industrialización y Políticas Económicas en Venezuela
}

Catalina Banko*

Resumen: En el presente artículo nos proponemos analizar el proceso de industrialización en Venezuela, tomando en cuenta las politicas adoptadas para promover este sector desde los años cincuenta y, de manera especial, a través del modelo de Sustitución de Importaciones en el siguiente decenio. Asimismo, se estudiarán los obstáculos confrontados por la industria en el transcurso de los años ochenta y, posteriormente, con la adopción del programa de estabilización macroeconómica en 1989 y el impacto de la apertura comercial en la producción manufacturera nacional.

Palabras-clave: Venezuela, Industrialización Sustitutiva, Proteccionismo, Mercado Interno, Competitividad.

Abstract: This article examines the industrialization process in Venezuela, taking into consideration the economic politics adopted since the 50's and, specially, the import-substitution industrialization model in the following decade. Furthermore, some emphasis is placed on the obstacles faced by the industry over the course of the 80 's and, later, with the neoliberal economic program and its impact on the national manufacturer production.

Keywords: Venezuela, Import-Substitution Industrialization, Protectionism, National Production.

\footnotetext{
* Profesora de la Escuela de Economía y de la Maestría de Historia de América Contemporánea de la Universidad Central de Venezuela. Investigadora del CELARG (1988-1992). Colaboradora de revistas especializadas nacionales y extranjeras. E-mail: catalinabanko@hotmail.com e catalinabanko@cantv.net. Recebido em 21/04/07 e aceito em 24/05/07.
} 


\section{Introducción}

A inicios del siglo XX, la economía venezolana estaba aún sustentada en los cultivos de café y cacao para la exportación. Debido a la especificidad de este género de explotación agrícola no se generaban encadenamientos productivos que permitieran propagar efectos dinamizadores en el conjunto de la economía y, al mismo tiempo, tampoco era posible aumentar considerablemente la producción debido a la restringida demanda mundial. Profundas deficiencias estructurales obstaculizaban el desarrollo agrícola y manufacturero, entre las cuales sobresalían: la segmentación de los mercados, la escasa circulación monetaria, la limitada inversión de capitales, las dificultades del transporte y el atraso técnico, a lo que se agregaba la ausencia de un sector capitalista sólido, la inestabilidad política y las recurrentes crisis económicas mundiales.

Se trataba de una economía que no generaba suficientes ingresos para ser dirigidos a la ampliación de la producción agrícola e industrial. Sin embargo, gracias a los efectos de la explotación petrolera en la expansión de los ingresos fiscales y del gasto público, comenzaron a manifestarse algunas transformaciones en el marco del aumento de la demanda de mercancías y servicios para el mercado interno, tendencia que fue tomando impulso a partir de la década de los cuarenta.

En el presente artículo nos proponemos analizar el proceso de industrialización en Venezuela, tomando en cuenta las políticas adoptadas para promover este sector desde los ańos cincuenta y, de manera especial, a través del modelo de Sustitución de Importaciones en el siguiente decenio. Asimismo, se estudiarán los obstáculos confrontados por la industria en el transcurso de los años ochenta y, posteriormente, con la adopción del programa de estabilización macroeconómica en 1989 y el impacto de la apertura comercial en la producción manufacturera nacional.

\section{Los primeros pasos en el camino hacia la industrialización}

A partir de 1936 se introdujeron importantes cambios económicos al conformarse un nuevo perfil de la acción estatal. La creación en 1937 del Banco Industrial de Venezuela favoreció el impulso de las manufacturas, 
aunque dirigido en su mayor parte al financiamiento de pequeñas empresas ${ }^{1}$. A través del Plan Trienal (1938-1941) se esbozaron los lineamientos del primer programa económico puesto en práctica en Venezuela, además de constituir el punto de partida de la aplicación concreta de políticas sociales, principalmente en las áreas de educación y salud.

Con base en la nueva concepción en torno al papel del Estado, el Plan Trienal ofrecía una propuesta de modernización económica mediante el fomento de la producción agrícola e industrial, objetivo que debía evolucionar en estrecha relación con el mejoramiento de las condiciones de vida de las "masas" y su educación. Se proyectó el fomento de ramas industriales destinadas al consumo interno para reducir así la salida de divisas en concepto de importaciones de artículos manufacturados.

Durante la Segunda Guerra Mundial, a consecuencia de la restricción de las importaciones y de la limitada oferta mundial de artículos manufacturados, se hizo perceptible la presencia de condiciones favorables para promover la industria. Paralelamente a la ampliación de las plantas fabriles ya existentes, fueron creadas numerosas empresas pertenecientes a rubros no explotados hasta esos años. A pesar de las dificultades derivadas de la escasez de maquinarias e insumos, el proceso de industrialización se extendió durante los años de la contienda mundial, gracias al aporte de créditos por parte del Estado y al incremento de las inversiones privadas.

Por entonces, se abrió una intensa discusión en torno a los alcances de la intervención económica del Estado y a la exigencia de implantar medidas de carácter proteccionista. En este contexto, se comenzaron a trazar políticas orientadas al fomento del sector industrial, conceptuado como el eslabón fundamental en la estrategia de modernización de la estructura productiva.

A juicio de Ernesto Peltzer (1965, p. 136-146), el principal objetivo de la industrialización debía estar encaminada a la mejora de la organización industrial y la elevación del rendimiento per cápita² ${ }^{2}$ Por tanto, estimaba conveniente utilizar el capital necesario para aumentar el rendimiento de las unidades productivas hasta el máximo que aconsejaran las condiciones generales de la economía, a través de un alto grado de capitalización por

\footnotetext{
${ }^{1}$ La mitad del capital del Banco Industrial de Venezuela fue suscrito por el gobierno y la otra mitad por accionistas particulares.

${ }^{2}$ Ernesto Peltzer se desempeñó desde 1940 como jefe del Departamento de Estudios del Banco Central de Venezuela.
} 
unidad de mano de obra, en vez de propiciar su "dispersión en multitud de establecimientos industriales".

En 1946 fue creada la Corporación Venezolana de Fomento (CVF), organismo destinado a otorgar financiamiento a las iniciativas privadas que pudieran contribuir al desarrollo de la producción interna tanto agrícola como industrial. Mientras se incrementaban las exportaciones petroleras, la economía experimentó un acelerado ritmo de crecimiento que se tradujo en la expansión de las inversiones privadas y públicas, así como también en la introducción de capitales extranjeros en agricultura y minería (CASTILLO, 1985, p. 36-46).

\section{Crecimiento acelerado del sector manufacturero}

En el contexto de las transformaciones económicas durante la segunda posguerra, la Organización de las Naciones Unidas promovió la creación en 1948 de la Comisión Económica para América Latina (CEPAL). Su primer estudio tuvo como objetivo el examen de la evolución de la economía latinoamericana desde los años treinta, lapso en que la industrialización se caracterizó por la lentitud de su crecimiento, limitado en gran parte a las ramas de textiles y alimentos (CEPAL, 1949, p. 1-18). A fin de imprimir mayor impulso a este proceso se consideró indispensable que el Estado asumiera un rol activo a través de la implementación de programas destinados a elevar la productividad agrícola e industrial e irradiar los efectos del desarrollo económico en el conjunto de la sociedad (CEPAL, 1949, p. 11-12).

Los lineamientos formulados por la CEPAL tuvieron gran influencia en diversos países del continente. En el caso venezolano, se observó la intensificación de la acción del Estado en materia económica, tendencia que se había iniciado, como ya lo señalamos, en el transcurso de la Segunda Guerra Mundial y prosiguió luego en los años de la posguerra. Precisamente, en la década de los cincuenta se puso en práctica el Nuevo Ideal Nacional, ${ }^{3}$ denominación que recibió la doctrina del régimen perezjimenista, que propiciaba el "reacondicionamiento gradual" del territorio nacional "en todos

\footnotetext{
${ }^{3}$ El Nuevo Ideal Nacional fue la doctrina adoptada por el régimen perezjimenista. Tras el golpe militar de 1948, el gobierno fue asumido por una Junta que en el año 1952 entregó la presidencia a Marcos Pérez Jiménez, quien fue derrocado el 23 de enero de 1958.
} 
sus aspectos físicos, para hacerlo más apto a la civilización contemporánea”. A partir de un esquema desarrollista y bajo un sistema político autoritario, se promovió la creación de sistemas de vialidad, infraestructura de uso agrícola, industrias básicas, electrificación, transporte y telecomunicaciones, obras turísticas, planes de vivienda, edificaciones escolares y unidades hospitalarias (CASTILLO, 1990, p. 157-159).

En el Nuevo Ideal Nacional se atribuía gran relevancia al desarrollo de la industrialización, que sería impulsada tanto por el sector privado como por el público. El Estado asumió la dirección de la petroquímica, siderurgia, teléfonos, electricidad y diversos servicios. También se proyectaron planes para incentivar la agroindustria, concretamente en los casos del maíz, arroz y azúcar, a fin de garantizar el abastecimiento de los principales productos de consumo interno. En un entorno económico de gran dinamismo, gracias a la expansión del gasto público y de las inversiones privadas, tanto nacionales como extranjeras, se manifestaron sustanciales cambios con el aumento de la población urbana y la ampliación de los estratos medios de la sociedad.

A fin de acelerar el crecimiento manufacturero, el Ministerio de Fomento creó en 1955 la Dirección de Planificación Industrial y Comercial para coordinar "el desenvolvimiento económico industrial y comercial, con la producción agrícola, pecuaria y minera”. La protección debía estar orientada al apoyo de las industrias de "mayor consumo de materias primas nacionales" o de productos "esenciales y de primera necesidad" (MINISTERIO DE FOMENTO, 1955, XVL).

El efecto dinamizador del conjunto de políticas aplicadas en esa década tuvo resultados alentadores en breve tiempo. Mientras el incremento del PIB fue de 9,3\% entre 1950 y 1957 , a la industria manufacturera le correspondió un crecimiento de 11,3\% (BCV, 1990). Si examinamos la evolución de algunas ramas industriales en el mismo período, podemos apreciar las siguientes tendencias: alimentos $8,8 \%$, bebidas $4,9 \%$, tabaco $4,7 \%$, artículos de cuero $11,8 \%$, industria de madera $10,8 \%$, textil $15,5 \%$, prendas de vestir $23,6 \%$, caucho $18,9 \%$, papel $31,2 \%$, maquinarias $5 \%$, minerales no metálicos $11,4 \%$ y productos metálicos 29,2\%. Las industrias básicas de hierro y acero, recientemente creadas, presentaron entre 1955 y 1957 un alza de 80,5\% (BCV, 1990). 


\section{El Estado "promotor" y el modelo de sustitución de importaciones}

En 1958, tras la caída del régimen perezjimenista, fue creada la Oficina Central de Coordinación y Planificación (Cordiplan), organismo que tenía como objetivo trazar los lineamientos de las políticas económicas para cada período presidencial. En el Plan Cuatrienal (1960-1964) ${ }^{4}$ se formuló de manera precisa el concepto de desarrollo económico que habría de materializarse mediante el impulso de la industrialización y modernización de la agricultura, todo ello bajo la acción promotora del Estado. Con relación a las manufacturas se propuso la aplicación de medidas de protección arancelaria y el otorgamiento de créditos al sector privado. Por su parte, el progreso de la productividad agrícola habría de lograrse mediante la asistencia técnica y financiera, paralelamente a la eliminación del latifundio, aspiración que formaba parte de la Ley de Reforma Agraria dictada en 1960.

El desarrollo industrial, según las argumentaciones del Ministerio de Fomento, se caracterizaba por sus efectos multiplicadores en la economía mediante la utilización de materias primas nacionales, tanto agrícolas como mineras, a la vez que extendía las oportunidades de empleo y contribuía a la elevación del ingreso nacional y a su distribución más equitativa. Se evaluaba que la industria contaba con algunos factores favorables: la existencia de un mercado interno que podría ampliarse progresivamente, la disponibilidad de capitales nacionales y extranjeros y de abundantes fuentes de energía. Sin embargo, era indispensable superar algunos obstáculos, tales como: la fuerte competencia de los productos extranjeros, la falta de un eficiente sistema de transporte, la escasez de mano de obra calificada y la inadecuada organización de gran número de empresas (MINISTERIO DE FOMENTO, 1959, p. 44-45).

Desde el año 1959, la Dirección de Industrias del Ministerio de Fomento se consagró a la tarea de promover dicho sector, considerado el factor clave para el "desarrollo económico" del país. Las políticas dispuestas a tal fin contemplaban el financiamiento a bajo costo, la asistencia técnica, la protección arancelaria y la exoneración de impuestos para la introducción de insumos y bienes de capital necesarios para la industria. Este apoyo se

\footnotetext{
${ }^{4}$ El Plan Cuatrienal (1960-1964) fue el primer plan económico diseñado por Cordiplan, organismo que fue creado en 1958.
} 
complementó con el otorgamiento de subsidios directos en algunos casos, como el de la industria lechera. Por su parte, la siderurgia y la petroquímica quedaron bajo la dirección del Estado, dado que se estimaba que los capitales privados no estarían en condiciones de afrontar las grandes inversiones requeridas para poner en funcionamiento dichas empresas (MINISTERIO DE FOMENTO, 1959, p. 498-499).

Como resultado de las medidas adoptadas se apreciaron signos favorables en breve tiempo. Entre 1961 y 1964, el Producto Industrial se elevó en $9,1 \%$, ocupando un lugar destacado los textiles: $13,2 \%$, prendas de vestir: 5,3\%, alimentos: 9,1\%, tabaco: 6,7\%, industrias básicas hierro y acero: $88,7 \%$ y materiales de transporte: $15,7 \%$. Poco después, la aparente pujanza de la industria comenzó a detenerse y, entre 1965 y 1969, su crecimiento fue solamente del 4.9\%. La situación específica de los rubros antes mencionados era la siguiente: textiles: $4.2 \%$, prendas de vestir: $-1,3 \%$, alimentos: $3,1 \%$, tabaco: $3,6 \%$, industrias básicas de hierro y acero: $10,9 \%$ y materiales de transporte: $8,5 \%$.

La desaceleración del ritmo de desarrollo industrial obedecía a las limitaciones del mercado interno, tanto por su reducido tamaño como por la desigual distribución del ingreso. Por otra parte, el proteccionismo, herramienta primordial para la etapa de despegue, se había convertido en un factor que, a mediano plazo, estaba frenando las posibilidades de expansión del sector, ante la ausencia de incentivos para multiplicar las inversiones y elevar la competitividad.

Cordiplan elaboró en 1966 la II Encuesta Industrial, en cuyos resultados se aprecia que la capacidad utilizada promedio de la industria fabril resultó ser de sólo el $60 \%$. En las ramas tradicionales ese aprovechamiento estaba restringido al 58\% y en las intermedias llegaba al 67\%. Las industrias mecánicas registraron una utilización de la capacidad instalada del 48\% (CORDIPLAN, 1970, p. 292-295).

Esta problemática fue analizada en el IV Plan de la Nación (1970-1974). El estancamiento de la evolución industrial fue atribuido a la persistencia de un modelo económico sujeto todavía a la utilización de la renta petrolera para financiar la inversión, a lo que se agregaba el deterioro crónico de los términos de intercambio. En este marco, el crecimiento económico no 
había incidido positivamente en la distribución del ingreso y de la riqueza social, marginando del mercado a amplios estratos de la población, con lo que quedaba conformado un "perfil de demanda de bienes y servicios poco propicio al desarrollo económico” (CORDIPLAN, 1970, p. 183-184). Estos conceptos reflejaban la preocupación gubernamental ante las limitaciones estructurales del modelo de industrialización diseñado, cuya reactivación sólo sería posible si se incentivaban las exportaciones. Precisamente, con el objetivo de extender los mercados se aceleraron las negociaciones para la integración de Venezuela al Pacto Andino.

Sin embargo, a pesar de las deficiencias evidenciadas en el proceso de industrialización, es menester subrayar su destacada contribución a la diversificación económica, además de los avances aportados en materia tecnológica, el aumento del empleo productivo y la capacitación de mano de obra. Según datos de Cordiplan, la industria manufacturera había acrecentado su participación en el PIB del 9\% en 1950 a 14,9\% en 1960 y al 20,2\% en 1969. Entre 1960 y 1969 las industrias intermedias pasaron de 31,6\% a $33 \%$, y en el caso de las mecánicas el incremento fue de $9 \%$ a $13,9 \%$. Sin embargo, todavía la integración del sector manufacturero no era catalogada como satisfactoria, ya que se requería de la importación de grandes volúmenes de insumos y bienes de capital, a la vez que las exportaciones de productos industriales no derivados del petróleo tenían escasa significación. A fin de corregir tales deficiencias, en el IV Plan se proyectó la conformación de una industria más dinámica, para lo cual sería "necesario el aumento del valor agregado a los productos fabricados en el país, mediante la producción de bienes intermedios y de bienes de capital y la concurrencia a mercados más amplios que el mercado nacional" (CORDIPLAN, 1970, p. 291).

En el marco de tales políticas, entre 1970 y 1973, el Producto Industrial subió en $7.5 \%$. Algunas ramas manufactureras presentaron en dicho período los siguientes índices: alimentos $6,3 \%$, textiles $6,6 \%$, prendas de vestir $2,7 \%$, tabaco $8.8 \%$ e industrias básicas $16,6 \%$.

\section{El proceso de industrialización en crisis}

La situación económica venezolana cambió de manera significativa a partir del boom petrolero de 1973 que posibilitó un incremento extraordinario 
de los ingresos fiscales. En este contexto, el V Plan de la Nación (19751979) proyectó ambiciosos programas para el desenvolvimiento del aparato productivo, principalmente en las áreas de petroquímica, siderurgia, aluminio y electricidad.

Con respecto a los objetivos del V Plan de la Nación, Purroy (1982, p. 284) señala que las inversiones públicas ya no eran de carácter complementario como en la década anterior, ya que el Estado se transformó "en el agente por antonomasia del proceso de acumulación". Bajo este esquema, la estrategia de desarrollo industrial se dirigió hacia la participación directa del sector público en la propiedad y dirección de empresas, el establecimiento de una nueva política proteccionista, el otorgamiento de incentivos fiscales para la producción interna y las exportaciones (CORDIPLAN, 1975, p. 116-118).

El Estado se propuso aportar el 53,1\% del total de las inversiones brutas, proporción muy superior al 31,9\% planteado en el IV Plan. Excluyendo la electricidad, el 94\% de las inversiones se centró en petróleo, siderurgia y aluminio (PURROY, 1982, p. 283-284). Entre las empresas que contarían con una elevada participación del Estado, se encontraban Sidor, Aluminios del Caroní (Alcasa), Venalum y el Astillero de Puerto Cabello. También se diseñó el programa metalmecánico para la rama automotriz y la fabricación de tractores agrícolas y motores (CORDIPLAN, 1975, p. 115-123).

Entre 1973 y 1976, la producción manufacturera subió en 11,1\%, gracias a las vastas inversiones públicas y a la mayor utilización de la capacidad instalada, al tiempo que la demanda interna subió a una tasa del 16\% anual. Esta brecha se amplió a partir de 1977, año en que el Producto Industrial aumentó en 4,8\% y la demanda interna en 21,4\%. En 1979 el 32\% de la demanda fue cubierta mediante importaciones. Nuevamente comenzó a detenerse el ritmo de crecimiento, ya que en el lapso 1977-1979, el Producto Industrial evolucionó a una tasa de 4,5\%. (CORDIPLAN, 1981, p. 104106).

Algunas ramas, con mayor capacidad para crear empleo, presentaron signos sumamente desalentadores, como es el caso de las fábricas textiles y de prendas de vestir que, entre 1977 y 1979, sufrieron una caída de 5,7\% y $3 \%$, respectivamente. Este fenómeno se explica por las importaciones indiscriminadas, dada la apreciación de la moneda venezolana y su elevada 
capacidad de compra en el exterior. Incluso, las industrias básicas de hierro y acero cayeron en $3.1 \%$ en los años mencionados, las cuales eran reputadas como uno de los puntales del programa de desarrollo.

Paradójicamente, en tiempos de "bonanza fiscal" se acentuó la vulnerabilidad de la economía venezolana cada vez más dependiente de las exportaciones de hidrocarburos. En la medida en que los recursos internos eran insuficientes para cubrir la magnitud de las inversiones proyectadas, fue necesario acudir al financiamiento externo. Inmediatamente, cuando los precios petroleros comenzaron a descender, quedaron al desnudo las limitaciones estructurales del modelo, ya que no era posible sostener planes de inversión con estancamiento del ingreso petrolero y creciente déficit en la balanza de pagos.

Las múltiples disposiciones de carácter proteccionista no parecieron tener efectos positivos y, más bien, se fue conformando una industria "ineficaz" que operaba con altos costos y no tenía capacidad exportadora. Desde el punto de vista del empleo, muchas de las industrias instaladas tenían un alto componente tecnológico por lo que absorbían una reducida proporción de mano de obra.

A pesar de los lineamientos establecidos por Cordiplan, no existían entes administrativos que coordinaran adecuadamente el desarrollo industrial. Con frecuencia se superponían las funciones de distintos organismos dependientes del Ministerio de Fomento y de Agricultura y Cría, por lo que se dispersaban muchas de las iniciativas adoptadas y no se alcanzaban los niveles de eficiencia indispensables y, menos aún, la adecuada integración entre ramas industriales (PURROY, 1982, p. 99-101).

Al iniciarse el gobierno de Luis Herrera Campíns (1979-1984), los síntomas del colapso del modelo rentista petrolero estaban ya aflorando con fuerza. La nueva administración planteó la necesidad de estimular la competencia mediante la eliminación de los controles de precios y la reducción de la protección arancelaria. A fin de corregir los desajustes económicos, se proyectó la reducción de la inflación a través de la contracción de la liquidez monetaria y la demanda interna, conjuntamente con el saneamiento de las finanzas públicas. Esta política se constituyó en el primer intento de implementar un plan de ajuste macroeconómico en Venezuela. 
Las medidas propuestas se aplicaron de manera desarticulada y fueron abandonadas a inicios de los ochenta, en el contexto de un transitorio pero significativo incremento de los precios petroleros. Sin embargo, hacia 1982 los desequilibrios se profundizaron ante la magnitud del déficit y la proximidad de los vencimientos de la deuda externa, cuyo peso se estaba convirtiendo en una carga insostenible, rasgo común en la mayoría de los países latinoamericanos. La fuga de divisas contribuyó entonces en forma determinante a empeorar la situación económica, que en 1983 desembocó en una grave crisis cambiaria, marcando el fin de la estabilidad monetaria que había caracterizado a la economía venezolana hasta ese momento.

La situación en los primeros años de la década fue dramática, tal como se observa en la evolución del PIB: 1980: -1,9\%, 1981: -0,3\%, 1982: 0,6\% y 1983: $-5,6 \%$. Por su parte, el Producto Industrial cayó también por primera vez en el año 1981 en 2\% y en 1983 en 1,6\%. En la rama textil y la de prendas de vestir se reflejó una baja del 5,1\% y del 3,1\%, respectivamente.

Esta declinación obedecía, no sólo a una crisis de carácter coyuntural, sino a la incidencia de diversos factores, entre los que sobresalían el exceso de protección y la escasa competencia que no se traducían en estímulos para la búsqueda de eficiencia y calidad en la producción. A ello se unían insuficiencias en el mercado de trabajo, un inadecuado abastecimiento de materias primas y otros insumos, el déficit de servicios e infraestructura pública y la débil "capacidad de gestión empresarial en algunos sectores" (CORDIPLAN, 1985, p. 107).

En medio de una profunda recesión económica, Jaime Lusinchi asumió la presidencia para el período 1984-1989. Este gobierno también se orientó en un primer momento al diseño de una política de ajuste para reducir el déficit mediante la contracción del gasto público. Sin embargo, era difícil atenuar los efectos de la crisis industrial, ya que tanto la inversión pública como la privada se redujeron significativamente entre 1983 y 1984, al tiempo que se originó un alza de los costos de producción a causa de la devaluación. Se dificultaron, además, los suministros oportunos de materias primas e insumos importados y surgieron dificultades de acceso a fuentes de financiamiento para la ampliación de las actividades manufactureras. Sin embargo, los programas sociales de carácter compensatorio y el plan 
nacional de mantenimiento de obras de infraestructura lograron neutralizar los factores que incidían en la reducción de la demanda agregada real (BCV, 1984, p. 156, p. 172-173).

Héctor Malavé Mata (2002, p. 29) señala que, desde los años ochenta, "el mayor riesgo de la economía venezolana consistía en su persistente subordinación a un modelo de crecimiento que no era capaz de forjar una acumulación autónoma sin el concurso del gasto público financiado con los aportes fiscales del petróleo". En ese marco, el modelo de Sustitución de Importaciones se convirtió en inviable, ya que cuando se dispuso de grandes recursos para el financiamiento de las inversiones industriales, la economía ya no tenía capacidad de "crecer sin importar cuantiosamente, ni capaz de exportar en la medida de contribuir a un balance comercial favorable".

Los desequilibrios económicos se fueron profundizando de manera acelerada, al tiempo que los intentos iniciales de aplicar programas de ajuste habían sido abandonados en 1986, con el propósito de poner en práctica planes de expansión del gasto fiscal. Debido a las cuantiosas erogaciones del gobierno, el Producto Industrial aumentó en 5,7\%, con una reanimación de la rama de textiles en $6,9 \%$ y la de prendas de vestir en 6,6\%, que anteriormente habían sufrido caídas pronunciadas. Sin embargo, el gasto público indiscriminado y el desorden administrativo durante el quinquenio presidencial de Jaime Lusinchi condujeron a un verdadero colapso económico, que en 1988 se tradujo en elevados niveles inflacionarios, caída de las reservas internacionales y agigantamiento del déficit fiscal.

\section{Estabilización macroeconómica y apertura comercial}

Frente a los alarmantes signos económicos, apenas iniciada la segunda presidencia de Carlos Andrés Pérez en $1989^{5}$, se decidió poner en práctica un nuevo programa de ajuste, después de haber suscrito una Carta de Intención con el Fondo Monetario Internacional para refinanciar la deuda externa. Con la nueva política adoptada, denominada "Gran Viraje", se pretendía disminuir el papel del Estado en la economía por medio de la liberación de precios y de las tasas de interés, junto a la instauración de la flexibilidad

\footnotetext{
${ }^{5}$ El presidente Carlos Andrés Pérez fue separado de su cargo en el año 1993 por decisión de la Corte Suprema de Justicia, asumiendo la presidencia provisional Ramón J. Velásquez hasta febrero de 1994.
} 
cambiaria del bolívar. Asimismo el gobierno se propuso reducir el déficit fiscal por medio de privatizaciones, el recorte del gasto público y la eliminación de subsidios y protecciones arancelarias al sector privado (MALAVÉ MATA, 1996). Se trató del tercer intento consecutivo - y el más importante - de imponer políticas de ajuste, las cuales habían sido rápidamente abandonadas en las administraciones anteriores.

El simple anuncio del nuevo plan económico y el aumento de las tarifas de los servicios públicos, especialmente de los precios de la gasolina, provocaron el estallido social del 27 de febrero de 1989. Este fue el primer llamado de atención a los partidarios del monetarismo. Debido a la reacción de la población, y por temor a futuras protestas, el gobierno puso en práctica de manera parcial algunas de las reformas propuestas.

En mayo de 1989 se estableció la normativa para la nueva política comercial de Venezuela, en la que se contemplaba la reforma gradual del arancel de aduanas. De acuerdo a tales disposiciones, se eliminarían los impuestos específicos y se fijarían aranceles que irían disminuyendo en los años sucesivos (LUCAS, 2006, p. 156).

Asimismo, se planteó con insistencia la aplicación de un programa de "reconversión" de las industrias para adaptarlas a las nuevas condiciones de la globalización de los mercados. Con tal fin, sería necesario poner en práctica un conjunto de "incentivos de carácter fiscal, tributario y financiero para apoyar aquellas empresas en proceso de reconversión” (LUCAS, 2006, p. 157). Sin embargo, tal propuesta debió enfrentarse a un cúmulo de obstáculos, ya que se trataba de una iniciativa que requería de inversiones extraordinarias para alcanzar adecuados niveles de competitividad en el mercado internacional. A pesar de que se creó el Fondo Venezolano de Reconversión Industrial y Tecnológica y fueron presentados diversos proyectos que apuntaban a tal objetivo, dicho programa no pasó del plano declarativo mientras persistían los signos de estancamiento.

En 1989, el PIB cayó en 8,3\% y el Producto Industrial en 14,4\%, lo que representó una de las crisis más profundas del sector hasta ese momento. Ello se reflejó en el caso de los textiles con una baja del 13,2\% y de 9,4\% en prendas de vestir. Otros rubros que hasta el momento se habían comportado de manera relativamente estable sufrieron también un fuerte impacto: 
alimentos: $-4,7 \%$, calzado: $-6,8 \%$, bebidas: $-18,3 \%$, industrias básicas de hierro y acero: $-11,2 \%$ y minerales no metálicos: $-17 \%$, entre otros.

Los datos precedentes evidencian el dramático desplome de la industria, a consecuencia de la caída de la demanda, altos índices de inflación, elevadas tasas de interés y alzas en los costos de servicios en general, a lo que se sumó el impacto de la introducción de multiplicidad de productos del extranjero. La situación se agravó por los recortes presupuestarios de PDVSA (la compañía estatal del petróleo) y las industrias básicas de Guayana, lo que incidió negativamente en la marcha de las empresas manufactureras privadas vinculadas con aquellas corporaciones. A ello se agregó la ausencia del tradicional apoyo del Estado en cuanto a las compras de artículos manufacturados venezolanos (CONSEJO DE ECONOMÍA NACIONAL, 1992, p. 20-21).

Después de un período de incremento de las exportaciones petroleras, se retornó a una situación de declive de los ingresos derivados de la explotación de hidrocarburos y, por tanto, del gasto público. Gerardo Lucas (2006, p. 161) calcula que entre 1989 y 1993 el Producto Industrial creció apenas en un promedio anual de 1,3\%. La apertura comercial tuvo graves efectos para la industria nacional, que ya venía experimentando sucesivas caídas desde 1980 .

Al respecto, Gustavo Linares Benzo (2003, p. 376-377) afirma: "La Administración eliminó los aranceles en productos manufacturados importados en un noventa por ciento, al mismo tiempo que los permisos especiales para la exportación, y simultáneamente reestructuró el sistema aduanero, disminuyendo los aranceles desde más de treinta y cinco por ciento en 1988, a menos de doce por ciento en 1992".

La situación de la industria no presentó síntomas de mejoría en los años posteriores. El control de cambios establecido entre 1994 y 1995 significó un duro golpe para las manufacturas que no pudieron resistir la competencia de las importaciones y el ascenso constante de los costos de producción. Por otra parte, las altas tasas de interés dificultaron el acceso a las fuentes de financiamiento, lo que generó la contracción de las inversiones. Todo ello estaba enmarcado, como agravante, en un clima inflacionario que afectaba profundamente a la industria, tanto por el aumento de los costos como 
por la disminución de la demanda. Como resultado de estas tendencias se exteriorizó, entre 1994 y 2000, una profunda crisis del sector industrial (LUCAS, 2006, p. 168-169). Gran número de empresas se vieron obligadas a cerrar sus puertas, mientras muchas de ellas eran absorbidas por firmas transnacionales. Prosiguió así el proceso de "desnacionalización, producto de la entrada de capital extranjero en forma de adquisiciones, fusiones, alianzas y asociaciones entre empresas" (MALAVÉ, 2003, p. 258).

La crisis industrial se caracterizó por la desaparición de gran número de pequeñas y medianas empresas que no estaban dotadas de tecnologías adecuadas para afrontar la competencia. La liberalización económica golpeó de manera contundente a la débil industria venezolana, con evidentes repercusiones negativas en el proceso de formación de capital y la capacidad de producción.

Humberto García Larralde (2001, p. 38, p. 45) afirma que el desempeño de la actividad manufacturera en la década de los noventa presentaba claros signos de crisis con bajos niveles de inversión y "creciente obsolescencia en equipos", factores que atentaban contra la pretendida competitividad del sector, al tiempo que se registraba un extraordinario incremento de las importaciones en el contexto de las políticas de apertura comercial y la consiguiente elevación de la competencia externa, a lo que se unían la sobrevaluación de la moneda y la disminución de la productividad.

\section{Conclusiones}

Desde su etapa inicial, el desenvolvimiento de la industria venezolana ha estado asociado a la acción promotora del Estado. Esta tendencia adquirió mayor fuerza a partir de los años cincuenta con el reforzamiento del papel empresarial del Estado. El gasto público se convirtió así en el motor de la economía, a la vez que la producción manufacturera llegó a tener una estrecha dependencia de la capacidad expansiva de dicho gasto. Mientras la industria estuvo orientada hacia el mercado interno, aunque limitado en tamaño y capacidad de consumo, logró crecer y alcanzar cierto desarrollo. Al modificarse las condiciones económicas internacionales, en el marco del proceso de globalización, ese sector no tuvo capacidad de adaptación frente a los nuevos requerimientos del mercado exterior, debido a su baja 
productividad, lo que generó la desaparición de gran número de empresas y la caída de la inversión privada.

El impacto de la liberalización económica fue demoledor para la industria venezolana, la cual había nacido y crecido al calor de la protección estatal. Paralelamente, las medidas de ajuste generaron una disminución de la capacidad adquisitiva y del consumo que se reflejó en la caída del producto industrial y en una elevada capacidad ociosa.

La problemática del sector industrial constituye motivo de honda preocupación por su estrecha vinculación con la creación de empleo y con la posibilidad de diversificar la estructura productiva y la generación de una economía menos dependiente de la renta petrolera. Asimismo, dicha actividad ha aportado a lo largo de muchas décadas una experiencia enriquecedora en materia de avances tecnológicos, formación de recursos humanos e incorporación de conceptos asociados a la idea de "sembrar el petróleo".

La recuperación de la industria venezolana implica la adopción de cambios estructurales en el contexto de un modelo de desarrollo económico sostenible en el tiempo, para lo cual se requiere redefinir el rol del Estado en la economía. En tal sentido, es fundamental el incremento de las inversiones, la elevación de la productividad, el logro de niveles aceptables de integración entre las distintas ramas industriales, la articulación de las acciones de los organismos públicos y la adopción de estrategias que, por un lado, superen las limitaciones del tradicional modelo sustitutivo y, por otro, tomen en cuenta las nuevas condiciones del mercado internacional. 


\section{Referencias Bibliográficas}

ABARCA, Karelys; MAYORCA, Eliana. Evolución de las formas de acumulación del capital en la industria venezolana durante la década de los noventa. Caracas, 2001. Tesis (Grado) - Escuela de Economía, Universidad Central de Venezuela, 2001.

ARAUJO, Orlando. Situación industrial de Venezuela. Caracas: Universidad Central de Venezuela, 1968.

BANCO CENTRAL DE VENEZUELA. BCV. Informe Económico. Caracas: 1984.

. Series estadisticas de Venezuela en los últimos cincuenta años. Caracas: 1990.

BAPTISTA, Asdrúbal. Un buen número: una buena palabra. Venezuela Siglo XX. Visiones y Testimonios, Caracas, Fundación Polar, 2002.

CASTILLO, Ocarina. Agricultura y politica en Venezuela 1948-1958. Caracas: Universidad Central de Venezuela, 1985.

- Los años del buldozer: ideología y política 1948-1958. Caracas: Universidad Central de Venezuela, 1990.

CEPAL. Estudio económico para América Latina. New York: Naciones Unidas, 1949.

CONINDUSTRIA. Directorio Industrial 2004. Caracas: 2005. Internet.

CONSEJO DE ECONOMÍA NACIONAL. Informe Anual. Caracas: 1992.

CORDIPLAN. Planes de la Nación. Caracas: 1960-1995.

GARCÍA LARRALDE, Humberto. Desarrollo industrial y tipo de cambio real. El caso venezolano. Revista de Economía y Ciencias Sociales, Caracas, n. 3, 2001.

INSTITUTO NACIONAL DE ESTADÍSTICA. INE. Indicadores de la fuerza de trabajo. Caracas: 2000-2003. 
LINARES BENZO, Gustavo. Regulación y economía: juntas y bien revueltas. Venezuela siglo XX. Visiones y Testimonios, Caracas, Fundación Polar, 2003.

LÓPEZ OBREGÓN, Clara; RODRÍGUEZ, Francisco. La política fiscal venezolana 1943-2001. Reporte de Coyuntura Anual 2001, Caracas, OAEF, 2002.

LUCAS, Gerardo. Industrialización contemporánea en Venezuela.1936-2000. Caracas: Universidad Católica Andrés Bello, 2006.

MALAVÉ MATA, Héctor. Las contingencias del bolivar. El discurso de la política de ajuste en Venezuela 1989-1993. Caracas: Fondo Editorial Fintec, 1996. . La trama funesta del petróleo. Nueva Economía, Caracas, n. 17, 2002.

MALAVÉ, José. El teatro de los negocios: formas, prácticas, actores. Venezuela Siglo XX. Visiones y Testimonios. Caracas, Fundación Polar, 2003.

MAZA ZAVALA, D. F. Cómo administrar contradicciones. Ajustes económicos en Venezuela. Nueva Sociedad, Caracas, n. 88, 1987.

MINISTERIO DE FOMENTO. Memoria. Caracas: 1948-1970.

MINISTERIO DE TRABAJO. Memoria. Caracas: 1960-1974.

OFICINA CENTRAL DE ESTADÍSTICA. OCEI. Indicadores de la fuerza de trabajo. Caracas: 1965-1999.

ORTEGA, Guillermo; NÓBREGA, Tobías. El epílogo de la Agenda Venezuela. Nueva Economía, Caracas, n. 12, 1999.

PELTZER, Ernesto. Ensayos de economía. Caracas: Banco Central de Venezuela, 1965.

PURROY, Manuel. Estado e industrialización en Venezuela. Valencia: Vadell Hermanos Editores, 1982.

RODRÍGUEZ CAMPOS, Manuel. Pérez Jiménez y la dinámica del poder (19481958). Caracas: Ediciones Eldorado, 1991.

SILVA MICHELENA, Héctor. Rumbos y contrastes del Plan 2001-2007. Nueva Economía, Caracas, n. 17, 2002. 
SUÁREZ FIGUEROA, Naudy. Programas políticos venezolanos de la primera mitad del siglo XX. Caracas: Universidad Católica Andrés Bello, 1977.

VALECILLOS, Héctor. Estadisticas socio laborales de Venezuela. Series históricas 1936-1990. Caracas: Banco Central de Venezuela, 1990. 